\title{
Culturas políticas y senderos pedagógicos: la reforma educativa inconclusa en Santa Fe (1983-2019)*
}

\author{
JUAN CRUZ GIMÉNEZ ${ }^{(1)}$ \\ MARCELINO MAINA(2)
}

Fecha de recepción: 30/03/2020

Fecha de aceptación: 31/07/2020

Resumen. El presente trabajo indaga el sistema educativo en la provincia de Santa Fe durante el período democrático pos 1983. Un sistema que se caracteriza por la persistencia de culturas políticas divergentes con una tradición de caducidad parlamentaria que resiste todo intento de reforma integral hacia un nuevo campo normativo pedagógico y educativo. El objetivo es identificar dos procesos reformistas en escenarios gubernamentales alternados entre oficialismo y oposición con un similar resultado: reformas educativas inconclusas. Investigar tensiones, impugnaciones, restricciones y actores gravitantes en clave de culturas políticas.

Palabras clave. Reformas educativas . culturas políticas $\cdot$ transiciones $\cdot$ decisionismo democrático
* Se exponen aquí algunas ideas debatidas, como miembros del Grupo Responsable, en el proyecto de investigación «Culturas políticas y discursividad. Santa Fe, I9I2-200I", que con la dirección de Bernardo Carrizo se lleva adelante en la Facultad de Humanidades y Ciencias, en el marco de la Programación CAI+D 2016 de la Secretaría de Ciencia y Técnica, UNL.

(1) Facultad de Humanidades y Ciencias, Universidad Nacional del Litoral (FHUC/UNL). Profesor y Licenciado en Historia (UNL), Magister en Ciencias Sociales (tesis en curso), docente de grado en la FHUC y de la FCJS/UNL, docente de área Investigación Educativa (Instituto Superior No 6) en Coronda, Santa Fe. Integrante de grupo CAID 2016. Coordinador junto a Bernardo Carrizo de Auroras en Provincia, Proyectos educativos $y$ discursos pedagógicos en la formación docente santafesina (1909 - 2009), Ediciones María Muratone, Santa Fe, 2011. Coordinador junto a Bernardo Carrizo de La política en las tramas educativas, Paraná, Del estante editorial, Editorial La Hendija, 20I7. cruzjuan74@hotmail.com

(2) Facultad de Humanidades y Ciencias, Universidad Nacional del Litoral (FHUC/UNL). Profesor de Historia y Magister en Ciencias Sociales (UNL), docente de grado en la FHUC/UNL. Coordinador de: Justicia y derechos humanos en la construcción de la democracia, Ediciones UNL, 2orr y autor de "Santa Fe» en Marcela Ferrari y Virginia Mellado (comps.), La Renovación Peronista: organización partidaria, liderazgo y dirigentes, I983-I99I, EDUNTREF, 20I6. Ha participado como coordinador en reuniones científicas; evaluador de artículos, jurado de concursos e integrante de comisiones evaluadoras, director y miembro de tribunales de tesis de posgrado y grado. Ha publicado artículos y participando activamente en la gestión de la FHUC/UNL. marcelinomaina@hotmail.com 


\section{Political Cultures and Educational Paths: the Unfinished Education Reform in Santa Fe (1983-2019)}

\begin{abstract}
This paper explores the education system in the province of Santa Fe, Argentina, during the post1983 democratic period. A system characterized by the persistence of divergent political cultures with a tradition of constitutional expiration that resists all attempts at comprehensive reform towards a new regulatory pedagogical and educational field. The objective is to identify two reform processes in governmental scenarios of
\end{abstract}

political alternation with a similar result: unfinished education reforms. This objective includes studying tensions, challenges, restrictions, and gravitating actors in terms of political cultures.

Keywords. education reforms · political cultures . transitions $\cdot$ democratic decisionism

\section{Introducción}

Desde fines de los años ochenta del siglo XX, la educación es un campo abordado a partir de una perspectiva interdisciplinaria en la que se cruzan aportes provenientes desde la historia, sociología, ciencia política e historia de la educación. Al mismo tiempo la educación es, en sí misma, un campo en términos analíticos, o sea, una «red o configuración de relaciones objetivas entre posiciones. Estas posiciones se definen objetivamente en su existencia y en las determinaciones que imponen a sus ocupantes, ya sean agentes o instituciones...» (Bourdieu y Wacquant, 1995:64). Como tal, el campo educativo nos permite poner en debate algunos temas, dilemas y perspectivas: instituciones, discursos, culturas políticas, imaginarios, reformas educativas, entre otros.

A partir de lo anterior, consideramos que la política y lo político atraviesan a la educación, además el vínculo entre lo nacional y lo provincial da cuenta de las condiciones multiescalares de los posibles abordajes del fenómeno educativo. En este trabajo nos centraremos en los procesos que reclaman su condición de pertenencia al entramado de las culturas políticas que participan de la invención de los diferentes itinerarios educativos que se definen en clave de políticas públicas. El terreno de la cultura política proporciona un abanico de matices que puede darnos las pistas para comprender la 
complejidad de los fenómenos sociales e indagar con otra perspectiva la dinámica de las instituciones. En el análisis de la cultura política, Héctor Schmucler señala que: "Hablar de innovaciones en la cultura política solo es posible pos facto, es decir, tras la verificación de una práctica. Si la cultura política no se agota en los lenguajes que la enuncian, la cultura política de un momento determinado es reconocida por los lenguajes que produce» (Schmucler, 1990:132-133).

Finalmente intentamos proponer y promover una consideración acerca de aquello que se denomina sistema educativo: entendemos que debemos pensar en la posibilidad de considerar la noción de sistema como un dilema. Más aún, proyectamos una mirada que instale la idea de lo educativo como una organización desorganizada puesto que de esa manera se ven incluidas dinámicas que rompen con la presunta secuencia lineal y homogénea de los procesos históricos. Incluímos la propuesta categorial de la incertidumbre y la complejidad como fenómenos matriciales de esta propuesta de análisis. Claramente desde la incierta transición democrática, y de allí en adelante, volvieron a ser revisados en clave educativa temas como la problemática estatal, la formación de la ciudadanía, la democracia y el autoritarismo en la educación, la gobernabilidad, entre otros tópicos como señala Laura Rodríguez (2013). En la década de los 90, este debate se vio fuertemente estimulado por la Reforma Educativa, que dio lugar a la generación de una importante cantidad de trabajos académicos a favor y en contra de ella, en una densificación del campo y en la consolidación de grupos de investigación. Los fenómenos propios de los años 80's ya mencionados, y los procesos de reforma neoliberal del Estado de los 90 (en especial de la educación y el agravamiento de la crisis de la educación pública), la aparición de la sociedad digital globalizada y las luchas de los grupos sociales por sus derechos demandaron la generación de respuestas y análisis que interpelaron a los historiadores de la educación.

Entonces, enfocando directamente el objeto de nuestro interés en el campo educativo durante la transición hacia la incierta democracia de los ochenta, un primer interrogante replica en cómo actuaron las culturas políticas luego del período dictatorial: los legados, las transiciones postergadas y las irresueltas. En ese registro, constatar que una prolongadísima temporalidad 
que comienza a fines de los años cincuenta con la sanción en 1949 de la última ley de educación provincial vigente en el distrito Santa Fe, señala la sedimentación de procesos, hitos, marcas y fenómenos culturales que atravesaron aquella legislación y con profundas mutaciones de aplicación y grandes vacíos de legislación, depositan la misma ley aún vigente en los albores de la tercera década del siglo XXI. Por lo tanto, entendemos que luego de la trágica experiencia del terrorismo de Estado, tanto en la Nación como en la provincia -recordemos la configuración constitucional federal de nuestro país- estaban en condiciones de debatir y proponer futuridades posibles; aunque -para nuestro caso en el plano educativo- se visibilizaba simultáneamente un «...cuadro donde se superponían los problemas introducidos por la experiencia autoritaria reciente con otros de más vieja data.» (Schmucler: 1990, 137-138)

Invocando el marco político, entendemos que es imprescindible considerar que desde 1983 la provincia fue gobernada por el Partido Justicialista hasta 2007 y desde esa fecha hasta 2019 por la coalición denominada Frente Progresista Cívico y Social. La hegemonía peronista durante los años 80's, 90's y primera parte del siglo XXI, tiene a su vez momentos e hitos interesantes de recuperar pues al menos hasta 1991-1993 la vida política estuvo dominada por una coalición de diferentes fragmentos del peronismo que intervenía en la designación de los cargos políticos y moldeaba el funcionamiento institucional en Santa Fe. Entonces, ¿qué aspectos deberíamos destacar de los dilemas que la transición proponía en términos culturales y educativos? A la cuestión de la imposición de una cultura del miedo, sumaríamos los derivados de la crisis económica de fines de los 70 y principios de los 80 , junto con las consecuencias del largo proceso de descentralización educativa que, activado a partir de la apertura política democrática en clave federal, generó dificultades importantes al momento de procurar, desde el ámbito nacional, construir políticas educativas de alcance general.

Si desde una perspectiva macro la construcción de un proyecto democrático se transformaba al calor de una verdadera explosión de civilidad en un futuro posible, en el espacio provincial santafesino, no ajeno a este clima de época, se generaba una situación clave en términos políticos: la provincia se transformaba en el bastión más importante de los sectores peronistas 
derrotados. Este dato definirá una relación progresiva y acentuadamente conflictiva con el gobierno nacional. Especialmente en 1980 se cierra una larga transición institucional que, comenzando a fines de la década del 60, se extiende hasta la definitiva desaparición de la Escuelas Normales en 1969. Y, a la par, se va operando lentamente en la confirmación de una especie de consenso tácito: la descentralización educativa. Así, la década del 80 comienza con una carga de sedimentaciones que remiten a transformaciones institucionales que, a caballo de una crisis global, marcará a aquella como un período de transición y, en consecuencia, de incertidumbre institucional en clave multiescalar nacional y provincial.

De este modo, consideramos que se refuerza la idea de cómo en el volátil escenario transicional que abarca los años 80's y 90's las culturas políticas heredadas de aquellos pasados-presentes se constituyen en contundentes desafíos para la construcción e invención de paradigmas societales democráticos, en particular hacia el interior del plano educativo. Desde la cima del Estado nacional y al interior de las experiencias subnacionales, la pregunta por la/s reforma/s adquirió alta contemporaneidad. En nuestro caso, la actualización del debate en torno a reformas educativas.

Entendemos que dos senderos se entrecruzan en ese recorrido: el primero, remite a las innovaciones a propósito del gobierno de la educación durante la gestión del gobernador José M. Vernet (1983-1987) y el derivado de las aspiraciones del II Congreso Pedagógico Nacional (1984-1988). El segundo deriva de una compleja y laberíntica transición que posicionó propuestas de reformas educativas que encontraron en el II Congreso Pedagógico Nacional su horizonte más ambicioso y el proceso que reveló la mayor parte de las tensiones corporativas encerradas en la discusión en torno a la educación. Los años ochenta y la invención democrática encuentran a las prácticas y representaciones educativas inmersas en un espiral acumulativo de tensiones que, traccionando las experiencias previas en una dinámica de centrifugación de los sentidos, promueve una dinámica de bloqueos corporativos y políticos que nos muestran a escala: política partidaria, legislativa, política corporativa (sindicatos, iglesia, etc.) e inclusive al interior del gabinete provincial: la constitución de un clímax de empate y de ausencia de una construcción discursiva y de políticas públicas educativas que se transforma en hegemónica. 
En el artículo aquí desarrollado nos detenemos en dos coyunturas dominadas por los vientos de reformas pedagógicas e institucionales en educación, ambas son pensadas como un necesario ejercicio reflexivo en clave de rupturas y continuidades para el caso santafesino. Los dos proyectos de reformas educativas impulsados desde el poder ejecutivo, liderando un proceso de aportes y debates para la construcción de consensos que en un caso (20052007) y en el otro (2016-2019) no lograron su cometido: una nueva ley de educación. Dos escenarios que remiten a culturas políticas heterodoxas pero atravesadas por una misma paradoja: la clausura electoral. Entonces, siendo esta la primera estación de este trabajo, y tomando como punto de partida los legados de los autoritarismos, emerge la apuesta por la invención democrática como horizonte de expectativas; pero al mismo tiempo, el peso específico de aquellos legados traducidos en la combinación compleja de varias culturas políticas de largo desarrollo, conducen a un callejón sin salida aparente para aquellos proyectos que ambicionan reformar los relictos dispersos de la educación provincial y, porque no, nacional.

\section{Reforma Educativa y Estado Provincial}

Desde aquellas consideraciones, se vuelve imprescindible pensar las iniciativas oficiales y el contexto de estrategia estatal que promovieron y promueven reformas en el campo educativo. Thomas Popkewitz ha llamado la atención sobre el significado y uso del concepto reforma en educación. En primer

(3) Popkewitz considera estos tres interrogantes: ¿En qué consisten las reformas educativas? ¿Cuáles son los diversos significados que adoptan con el paso del tiempo? ¿De qué manera se elaboran esos significados como elementos de relaciones de poder? Los discursos de las reformas educativas aparecen aquí investigados desde un punto de vista temático más que cronológico. lugar, en tanto la historicidad del concepto tiene dinámica propia, allí apela a una ecología de las reformas en educación donde el ambiente institucional es clave. ${ }^{(3)}$ Dicha ecología, está determinada por las pautas de regulación social de la escolarización. Una afirmación de ingreso: los alcances de una reforma no se reducen a una ley o proyecto de ley. Siguiendo a Popkewitz la referencia es a un proceso que emerge en sus dimensiones. A saber: Utilizo aquí el concepto de estado para referirme a algo más que a la política gubernativa o la legislación oficial. Se usa como una categoría teórica para explorar cómo se aplicaron las estrategias para construir la reforma, las categorías y distinciones empleadas para describir los 
fenómenos escolares, y cómo los contextos sociales de la formación del profesorado y de la escuela interactúan en tanto que prácticas de gobierno que producen valores sociales y relaciones de poder (Popkewitz, 1994:76)

En segundo lugar, las distinciones y categorías que subyacen a las prácticas educativas no son términos neutrales y descriptivos; la amalgama de acciones y de prácticas discursivas se interpreta como un tipo particular de conocimiento históricamente elaborado en el que se inscriben ciertas formas de actuar, sentir, hablar y ver el mundo. Estudiar la reforma escolar es interpretar cómo las categorías y los propósitos políticos van disciplinando y configurando nuestros sentidos de la elección y la posibilidad. Los debates parlamentarios son comprendidos como escenarios de disputa de proyectos formulados para la regulación estatal de la educación. Aquí la motivación por definir un primer enfoque de aproximación a saberes especializados, la agencia estatal y las interpretaciones dominantes que interpelan a las prácticas educativas en la provincia durante los años del retorno democrático en las administraciones justicialistas (1983-2007).

Como antecedentes, es posible afirmar que la educación santafesina, a diferencia de otros escenarios provinciales o regionales, se caracteriza por una constante: la ausencia de reformas integrales desde mediados del siglo $X X$. $^{(4)}$ El campo educativo provincial está regulado, tal como mencionamos más arriba, por la ley N03554 de agosto de 1949 en forma continua hasta nuestros días. Dicha norma, consecuencia de la máxima armonía hegemónica del peronismo santafesino luego de la reforma constitucional de 1949 (con mayoría parlamentaria) reemplaza la ley escolar de $1886 .{ }^{(5)}$ Hay que tener en cuenta que la ley 3554 fue derogada y suspendida por el decreto ley 8199 de 1956 como consecuencia de la etapa de proscripción que caracterizó al posperonismo luego de la Revolución Libertadora (1955) sin ser reemplaza hasta hoy por una nueva norma integral. Esta novedad aparentemente menor, se contrapone al resto de los Estados provinciales en Argentina que durante la década de 1990 encontraron normas sancionadas y aprobadas para la regulación de la educación, acompañando el proceso de reforma liberal del campo educativo a partir de la ley nacional conocida como Ley Federal de Educación. ${ }^{(6)}$
(4) Desde el campo especializado, Thomas Popkewitz (1994) ha llamado la atención sobre las reformas en educación.

(5) La tradición normativa y reformista sobre el gobierno de la educación en la provincia de Santa Fe es un campo fértil para nuevos estudios provenientes de perspectiva de historia regional de la educación. No abundan los trabajos académicos en esta materia, tarea que compartimos Bernardo Carrizo, Marcelino Maina, Juan Cruz Giménez en conjunto con colegas docentes en archivos institucionales y equipos de investigación. Consecuencia de dicha motivación resultó la publicación Auroras en Provincia (2010) y la necesidad de ampliar la escala de observación, el trabajo en archivos, los diálogos académicos y el intercambio de experiencias relativas a pensar el gobierno de la educación en esta clave.

(6) La tradición hegemónica $\mathrm{y}$ académica dominante en el mundo anglosajón durante los 80 y 90, de impronta estructuralista ha impactado en el escenario de Argentina en los últimos ańos; cabe citar el trabajo dirigido y coordinado por Axel Rivas (CIPPEC, 2008, 2010) como resultado de un extenso y profundo trabajo publicado como: Gobernar la educación; Estudio comparado sobre el poder y la educación en las provincias argentinas. 
(7) Se especifica que la regulación estatal sobre el sistema educativo en su totalidad contempla un conjunto complejo de aristas sobre los actores que integran dicho sistema en sus roles y funciones. En Santa Fe, las leyes vinculadas al campo educativo y sancionado durante la década de 1990 solamente se relacionan a la regulación de aspectos laborales (incompatibilidades, régimen de disciplina docente, jubilación del docente y licencia por maternidad). Por lo que no se analizan aquí como norma integral que regule al sistema en su conjunto.

(8) La ausencia de una normativa que regule el funcionamiento de un Consejo Provincial de Educación en el caso analizado es un indicador que deja al descubierto la inexistencia de instancias colegiadas y resolutivas propias al campo educativo. La gestión territorial (de la educación provincial) se divide hasta la actualidad por decreto del poder ejecutivo en nueve direcciones regionales (decreto 456 de 1986), y regula roles, misiones $\mathrm{y}$ funciones de equipos supervisivos y directivos.

Mientras que el decreto 4597 de 1983 reglamenta licencias, franquicias y justificaciones para el personal docente $y$ auxiliar docente. La ley de disciplina para el personal docente fue sancionada en 1988 .

(9) Durante 1994 se sanciona la ley de incompatibilidad docente (II237) vigente en la actualidad.

\section{Transición y reformas inconclusas}

El acceso a los archivos disponibles en el período que nos ocupa, da cuenta de la abundante existencia de proyectos de ley para regular la educación en la provincia: más de una docena de proyectos se encuentran archivados en la categoría «caducados». Categoría que indica la pérdida de estado parlamentario y la consecuente ausencia de norma mayor; como contrapartida, son varios los instrumentos reguladores, como: decretos, resoluciones y digestos que orientaron las conductas de los actores del campo educativo desde el retorno de la democracia. ${ }^{(7)}$

Recuperando algunos planteos, cuando se piensa en el marco político santafesino lo primero a señalar es que, desde el regreso de la democracia, la provincia ha sido gobernada ininterrumpidamente por el Partido Justicialista. A pesar de la hegemonía peronista en este período (24 años), un corte muy claro puede establecerse entre las décadas del 80 y del 90 en tanto transiciones en tiempos de democracia. Durante el primer período, la vida política estuvo dominada por una coalición de diferentes fragmentos del peronismo que intervenía en la designación de los cargos políticos y controlaba el poder de los dos gobernadores que se sucedieron: José María Vernet (1983 - 1987) y Víctor Reviglio (1987 - 1991). El sistema educativo se reguló por decretos en función de prioridades: el ordenamiento territorial y administrativo, facultades jerárquicas de equipos supervisivos y de conducción escolar, la regulación de licencias laborales y una ley relacionada con la disciplina para el personal docente. ${ }^{(8)}$

A partir 1992 se inicia un decisivo segundo momento durante las administraciones de Carlos Alberto Reutemann (1991-1995 y 1999-2003) y luego la continuidad gubernativa del justicialismo con el gobierno de Jorge Obeid, también en dos oportunidades (1995-1999 y 2003-2007). (9) En el marco de la contextualización del campo educativo, es necesario plantear el mapa de los actores que intervienen en la formulación e implementación de las políticas públicas educativas. El hecho que en Santa Fe, a diferencia de otras provincias, no exista un órgano colegiado como el Consejo de Educación -o figura similar- deja en manos de un solo organismo -el Ministerio de Educación- el gobierno y la gestión de la educación, otorgándole al Poder Ejecutivo un rol central en el desarrollo de las políticas educativas. ${ }^{(10)} \mathrm{Al}$ 
contrario, el Poder Legislativo se caracteriza por su debilidad, su dependencia del Ejecutivo y la escasa autonomía propia a la hora de regular la educación. Durante esta primera etapa de gobiernos justicialistas y coaliciones cuyo eje era el peronismo, los perfiles de los ministros de educación elegidos respondieron a un impulso de reordenamiento del sistema: fueron todos abogados o ingenieros con poca o nula experiencia en el ámbito educativo, pero con una fuerte impronta en la negociación y la administración eficiente. El primero de los ministros, Danilo Kilibarda (ex integrante de la Comisión de Educación de la Cámara de Diputados de la Legislatura provincial), realizó un diagnóstico detectando las «zonas oscuras» del Ministerio y dio los primeros pasos hacia el reordenamiento, entre otros, modificando la estructura del organigrama. ${ }^{(11)}$

Luego de una breve experiencia de Ricardo Kauffman en la cartera educativa, la gestión iniciada por Fernando Bondesío siguió un plan de eficiencia. El nuevo ministro, partiendo de una firme convicción sobre las modificaciones que debían realizarse y contando con un fuerte apoyo político, logra por fin introducir varias medidas duras y dolorosas en pos del reordenamiento de distintos aspectos del sistema y del fin de los paros docentes.

La primera de estas conquistas se plasmó en la profundización de la regionalización, la suspensión de las comisiones de servicio -espacio destinado por excelencia a otorgar cargos públicos sin demasiada justificación-, el traslado del control de las licencias del personal docente bajo la órbita del Ministerio de Educación -que antes estaban bajo dependencia del Ministerio de Salud- y el reordenamiento de los subsidios otorgados a los establecimientos de gestión privada. La segunda, sostenida por el cansancio de las familias que reclamaban la asistencia regular de los maestros a las escuelas, fue la consecuencia de la introducción del presentismo en el salario docente, medida que modificó sustancialmente las relaciones entre los gremios y el Estado. ${ }^{(12)}$ El acceso al archivo de la legislatura de la provincia de Santa Fe permite encontrar los proyectos de ley «Regulando el sistema Educativo en la provincia» en su situación de «caducados». Por mencionar solo un caso,
(10) El entonces subsecretario de educación, el profesor Bernardino Turri, define clara y duramente la evaluación que se hacía del sistema en el Ministerio: «...el sistema educativo está roto, dańado por todo tipo de anormalidades (...). A medida que avanzamos vamos notando las profundas deficiencias en la faz organizativa (...). El docente, además de sus problemas económicos, estaba tapado por una maraña de situaciones irregulares. Nuestra primera tarea, en consecuencia, es ordenar el sistema, tratando de que cada uno de sus integrantes cumpla con su función (...). Hay que desmalezar, recrear el servicio. " Sin embargo, la gestión de Kilibarda finalizó antes de cumplir tres meses por "diferencias de estilos» con el gobernador, respecto de la modalidad de negociación gremial.

(11) Ver la propuesta de Mariano Sironi (UNR) sobre «El rol del Estado en el ámbito educativo en la provincia de Santa $\mathrm{Fe}$ » analiza las tensiones propias del proceso de descentralización en el ámbito educativo en la provincia de Santa Fe durante los años 1987-1995. Investigando las dimensiones vinculadas a la matriz estadocéntrica y las reformas neoliberales en dicha escala.

(12) En este marco, y teniendo en cuenta la escasez de proyectos de ley propuestos por la legislatura comparada a la superabundancia de decretos y resoluciones, la tarea del legislativo parece especialmente dedicada a influir sobre el Poder Ejecutivo 
para lograr que «vea con buenos ojos» (tal es la fórmula que introduce las demandas) la satisfacción de los pedidos del territorio representado.

Así, la atención particular prestada, especialmente por los senadores, a las solicitudes (más o menos justificadas) de edificios, cargos, infraestructura, material didáctico, surgidas en los departamentos que representan, parece caracterizar su función. Al respecto, ciertas visiones críticas insisten sobre las distorsiones que crea en el conjunto del sistema este modo de gestión de las necesidades.

(13) Además de Rébola se pueden mencionar los proyectos presentados por los diputados Sánchez (2000),

Gutiérrez (1998), Favario (1995), Robles (1993), Cantero (2006), Marcucci (20I0) entre otros.

(14) Adriana Cantero asumió como Ministra de Educación de la Provincia de Santa Fe en octubre de 2005 como reemplazo de su antecesora

Carola Nin. El entonces gobernador Jorge Obeid tomó la decisión en un escenario de conflicto con el gremio docente luego de 24 días con medidas de fuerza. La gestión de Cantero, aunque sostenida

hasta el final de mandato de Obeid, estuvo atravesada por resistencia gremial del sur provincial, la crisis con el referente de Educación

Técnica prof. Fernando Pisani y fuertes críticas por la demora en la implementación de programa educativo en materia de Educación Sexual, acompañada por acciones el diputado Alejandro Rébola ingresa proyectos del mismo tenor en 1996, 1998 y 2000 . $^{(13)}$

Consideramos importante, a continuación, hacer referencia a la coyuntura reformista convocada desde el Ministerio de Educación a cargo de Adriana Cantero, parte del equipo del ejecutivo provincial a cargo de Jorge Obeid en su segundo mandato. ${ }^{(14)}$ El proceso de convocatoria para el debate, aportes y consensos a docentes, equipos directivos y supervisores se puso en marcha desde junio de 2006 a propósito de la agenda propia de la cartera educativa nacional en función de lograr consensos para un nuevo cuerpo normativo que deje atrás la Ley Federal de Educación de 1995. El 5 de julio de 2006 se llevó adelante una jornada nacional de trabajo sobre estos temas:

En la jornada nacional se propone un documento donde estarán los principios de la educación actual, es un debate abierto, donde las escuelas producirán un informe, que se elevará al supervisor, él elaborará un resumen de las tendencias, y nosotros vamos a trabajar en cada regional, para hacer un informe regional que será elevado a Buenos Aires. Tenemos que pensar que una ley siempre es una ley en perspectiva, se piensa en una apuesta a futuro. Tenemos que pensar qué otras cosas queremos que constituyan en una meta para la educación argentina. ${ }^{(15)}$

El clima de reforma educativa en nación como en provincia busca resultados concretos en plazos inmediatos. En nación, la etapa coordinada por Daniel Filmus como ministro del gobierno de Néstor Kirchner está atravesada por una nueva contienda electoral que tendrá consecuencias directas en el proceso de consultas iniciado. En la provincia de Santa Fe, Adriana Cantero socializa una cruzada con expectativas fundantes, pues para la ministra la coyuntura que inaugura la primera década del siglo XXI constituye una oportunidad única para una reforma profunda y estructural de la educación provincial. En un artículo de opinión, la funcionaria de la cartera educativa realiza un detallado análisis del escenario santafesino que da cuenta la sanción de un nuevo marco normativo nacional (la ley de Educación N²6.206): 
La Argentina ha comenzado a transitar un tiempo de transformaciones estratégicas en su sistema educativo: nueva estructura, más modalidades, expansión del nivel inicial y también de la educación secundaria, fortalecimiento de la educación técnico-profesional, creación de un sistema de formación docente que incluye institutos superiores y universidades, extensión de la obligatoriedad de cursado. (Cantero, 2008:12)

El horizonte de la reforma educativa nacional, con una fuerte crítica a su normativa anterior (Ley Federal de Educación de 1990) se replica en el escenario provincial a partir de indicadores que Cantero (2008:22) identifica como insuficientes y necesitan una adecuación a los desafíos predominantes: «Pensar en una educación obligatoria de trece años nos obliga a mirar las trayectorias escolares de nuestros niños y adolescentes hoy, con un trayecto con idéntico mandato de solo diez años y que aún no hemos logrado cumplir».

Otro punto bisagra, al que Cantero (2008:22) presta atención en el proceso de reforma está en el $9^{\circ}$ año del sistema o el puente entre el nivel EGB 3 y el ciclo Polimodal, como profunda barrera de acceso y continuidad:

Por otra parte, los operativos de evaluación indican niveles de desempeño que ponen en cuestión la calidad de los procesos de enseñanza y aprendizaje en la escuela. Señalan claramente que el $9^{\circ}$ año de EGB en 2005 sigue siendo un año significativamente vulnerable y, en tanto «puente» hacia el ciclo orientado de la educación media, condiciona la posibilidad del acceso a conocimientos más complejos. Pero además, si bien con mejores resultados, los aprendizajes básicos tampoco se logran en los años iniciales de la escuela primaria con la claridad debida.

El sistema educativo integral en su nivel secundario presenta dificultades a superar desde una mirada sistémica. En este sentido, Cantero afirma:

Diseñar una escuela secundaria pensada para los adolescentes y para enfrentar la masividad del alumnado nos pondrá en situación de definir nuevas propuestas curriculares (será necesario analizar críticamente el ciclo orientado y los sistemas de evaluación). Requerirá además un trabajo particularmente destinado a recuperar la unidad pedagógica, y el esfuerzo por determinar una organización que permita: reconfigurar los puestos de respaldo a la jerarquía eclesiástica determinante en el campo educativo provincial. Ley de Educación Sexual No I0.947, provincia de Santa Fe, 1992. Obsérvese que durante el año 2005 se modifica la ley de jubilaciones del personal docente ( $\mathrm{No}^{\mathrm{I}} 2464$ ) o «nueva ley de jubilaciones».

(15) El Litoral, «Día histórico para la docencia argentina», entrevista a la ministra Adriana Cantero, Santa Fe, 05/07/2006. 
(16) «Una política de Estado para la Educación Santafesina.

Documento para el debate», Ministerio de Educación, Gobierno de la provincia de Santa Fe, julio de 2007, imprenta oficial. Entre los desafíos se distinguen el ingreso, la permanencia,

la formación y el egreso de alumnos en sus distintos

niveles.

(17) El Litoral, (26/11/2007)

p. 4 de trabajo, generar nuevos roles y funciones, fortalecer las tutorías y los dispositivos de apoyo, planificar acciones de seguimiento del alumnado. Se hace indispensable planear la ampliación de la cobertura mirando zonas rurales y urbanas más vulnerables y atender al mismo tiempo la necesidad de mejorar la relación matrícula-docentes en las localizaciones superpobladas, así como también en las más carentes de profesorado (Cantero, 2008:23).

El Ministerio propone para la consulta, análisis y aporte un documento titulado «Una política de Estado para la Educación Santafesina», que puede ser útil para la comprensión del contexto y de los fundamentos de la futura norma. De este modo, con la consulta 2007 se renueva la participación, se profundizan los debates, se afinan los consensos y la provincia de Santa Fe aparentemente se encamina a su ley de Educación. ${ }^{(16)}$ Dicho documento consta de 10 apartados, presenta estadísticas sobre las trayectorias educativas e identifica desafíos de la realidad educativa. Durante el año 2007 se llevaron adelante elecciones nacionales y provinciales. En la provincia de Santa Fe, la convocatoria a las urnas definió el final de una larga hegemonía ininterrumpida del peronismo provincial en el ejecutivo provincial luego del retorno a la democracia en 1983.

En este contexto de transición, la ministra Cantero se fijó como meta dejar preparado el proyecto de ley educativa provincial, y así lo hizo. Con resistencia en varios frentes, se dio a conocer en las escuelas el documento titulado «Una política de Estado para la educación santafesina. Síntesis final». El Litoral en su edición del día 26/11/2007 da a conocer la novedad:

....recién salida de la imprenta oficial, la publicación contiene una presentación donde asegura que el proyecto de ley es producto del debate con docentes y distintos protagonistas del sistema educativo. Luego expone una síntesis de lo discutido en la Mesa Ampliada con los representantes de la comunidad y, finalmente, presenta el proyecto per se con sus 164 artículos.» El documento síntesis final se organiza en partes. Como epílogo, se asegura que el escrito podrá «ser corregido, mejorado y rediscutido» dado que la pretensión es «señalar un rumbo, establecer bases para una política de Estado». ${ }^{(17)}$

En la transición gubernativa y política, el equipo de gestión entrante de Hermes Binner con Élida Rasino como ministra de Educación, solicitó a la 
gestión saliente de Obeid que no envíe a la Legislatura ningún proyecto de ley educativa. ${ }^{(18)}$ En ese mismo sentido, se pronunció el gremio AMSAFE -de la educación pública- que no participó de la convocatoria de Cantero a debatir sobre la futura ley en la Mesa Ampliada. Solamente envió un documento crítico y algunas líneas de reflexión, pero no estuvo presente en las rondas de consulta. Sobre el contenido elaborado por la (por entonces) ex ministra Cantero, el proyecto no define a la educación como un servicio público esencial, una cuestión que ponía en alerta al sindicato docente que ve en esa concepción una restricción al derecho a huelga. Sin embargo, introduce otras cuestiones que generan controversia. Por ejemplo, la idea de incluir a la familia como "agente natural y primario» de la educación, y de considerar entre los fines de la enseñanza la formación integral de las personas, que incluye la «dimensión religiosa». Estos conceptos son vistos por algunos sectores como un avasallamiento a la educación laica. El proyecto propone, además, la creación de un Consejo Consultivo de la política educativa, y promueve que las escuelas privadas que atienden a la población de menores recursos sean incluidas en los aportes regulares que el Estado provincial destina, por ejemplo, a mantenimiento e infraestructura.

\section{Decisionismo democrático y nuevos intentos de reformas}

En este punto, haremos referencia a la segunda etapa de una reforma inconclusa (el proyecto de ley de Educación que obtuvo media sanción en la cámara de diputados de la provincia (y una notable resistencia y rechazo en la cámara de senadores). Las experiencias del Frente Progresista y las fuerzas aliadas que desplazan al justicialismo de la casa gris son tres: Hermes Binner (2007 - 2011), Antonio Bonfatti (2011 - 2015) y Miguel Lifschitz (2015 - 2019). . $^{(19)}$

Durante las administraciones de Binner y Bonfatti no fue posible ni prioritaria la promoción de reforma legislativa alguna sobre el campo educativo. En este sentido, los cuadros de gestión educativa participaron orgánicamente del Consejo Federal de Educación e innovaron en programas y ejes de acción que materializaron en las escuelas y las comunidades educativas un aire de
(18) «Enviamos el documento a la Casa de Gobierno, y lo estamos repartiendo en las escuelas y a los miembros de la comunidad que participaron del debate. También se lo remitiremos a los legisladores y a las autoridades entrantes", señaló a El Litoral la ministra Cantero. Consideró que el texto de ley "es un muy buen trabajo" y evitó opinar sobre si Jorge Obeid debería enviar el mensaje a la Legislatura para su tratamiento. «Es una decisión que tiene que tomar el gobernador", se limitó a decir. De no hacerlo, señaló que el trabajo no habrá sido en vano puesto que «la reflexión y la participación son marcas que quedan en los actores, que son los mismos que supuestamente tendrán que producir otro trabajo». Binner aprobó la vigencia en sus primeros días de gestión de la ley No I2799 que ordena al personal docente en tareas diferentes, transitorias o definitivas.

(19) Las ministras de educación durante las administraciones del Frente Progresista a partir del ańo 2007 fueron: Élida Rasino (gobierno de Hermes Binner), Leticia Mengarelli (durante Io meses) y Claudia Balagué (gestiones de Antonio Bonfatti y Miguel Lifschitz). 
(20) Entre las medidas innovadoras se destacan: ley de paritarias docentes (I2.958/2008), ley comités mixtos para la docencia (I2.913/2008), régimen de convocatorias para concursos y titularizaciones, la jerarquización del área de Bienestar Docente a cargo de Leonardo Panozzo, leyes modifictorias al régimen de jubilación docente. Además de la aprobación de una secuencia de decretos como: decreto 257/2008 que determinó la finalización de exigencias de postítulos en la formación superior como consecuencia directa de la aplicación de la ley de Educación Superior; decreto 2I28/20I3 reglamentación de la carrera para docentes de educación artística; creación de juntas de escalafonamiento por decreto I339/2008; reorganización y modernización de procedimientos administrativos por decreto 4I7I/2OI2; decretos de franquicias por maternidad (I494/2009 para docentes de nivel inicial y 4192 para modalidad especial; decreto 2703/2010 integración institucional NNyA con discapacidad.

(21) La aprobación del decreto 2288/2016 conocido como Protocolo para el abordaje institucional de acceso a la justicia de NNyA víctimas de delitos y abusos fue un documento que entró en vigencia en la etapa de Miguel Lisfchitz. primavera. ${ }^{(20)}$ Un repaso somero del conjunto de medidas normativas -en su mayoría decretos- fija prioridades en la ejecución de políticas educativas en cada etapa de gobierno. En el caso de Hermes Binner, regulaciones para la institucionalización del Bienestar Docente y una recuperación de la tarea docente en un proceso progresivo de acciones y medidas que clausuren la etapa de reformas neoliberales iniciadas durante la década de 1990. Las adecuaciones normativas de la gestión de Antonio Bonfatti reordenaron el sistema único de reglamentación de la carrera docente -a través decreto 3029/2012- y cambios en la ponderación de antecedentes profesionales. Durante 2016 el gobernador Miguel Lifschitz anunció la intención de elaborar una ley educativa provincial.. ${ }^{(21)}$ Así, en la Cámara de Diputados se presentaron cuatro iniciativas. La propuesta de Alejandro Boscarol (UCR Frente Progresista Cívico y Social) impulsa la obligatoriedad del nivel inicial desde los tres años, la autoevaluación y la creación de consejos escolares. Además, establece que se destine a la educación un porcentaje de recursos no inferior al 35 por ciento del presupuesto anual. Por otra parte, los diputados Rubén Giustiniani y Silvia Augsburger (Igualdad y Participación) presentaron dos iniciativas. Allí se establece que la educación pública es gratuita, laica, igualitaria y obligatoria desde los tres años hasta finalizar el secundario. Además, establece como derecho de los estudiantes, recibir educación sexual integral, desarrollar los aprendizajes en edificios que respondan a normas de seguridad y participar en la elaboración de las normas de convivencia escolar. Por su parte, Verónica Venas (Pares, FPCyS) presentó un proyecto para crear la Universidad de Santa Fe, organizada en base a los institutos de formación superior de la provincia. Del mismo modo, los diputados Carlos del Frade y Mercedes Meier (Frente Social y Popular) hicieron su propuesta. Entre otros puntos, el proyecto establece la obligatoriedad del nivel inicial desde los cuatros años y la transferencia progresiva de las escuelas privadas subvencionadas por el Estado. Además, busca garantizar un porcentaje de recursos no inferior al 40 por ciento del presupuesto anual de la administración provincial.(22)

Una síntesis de los proyectos presentados en la cámara de diputados fue posible a mediados de 2018 , incluso la apertura legislativa a especialistas permitió la incorporación de un documento base para la discusión a cargo 
de la Universidad Nacional del Litoral. Equipo integrado por Investigadores de la Facultad de Humanidades y Ciencias (FHUC), junto con especialistas de otras facultades de la Universidad Nacional del Litoral (UNL) y de la Universidad Nacional de Entre Ríos (UNER), presentaron un dossier con aportes para su tratamiento a los integrantes de la Comisión de Educación de la Cámara de Diputados provincial. En el mismo sentido, tuvieron la voluntad de hacer lo propio con los senadores provinciales. La dinámica de trabajo en la UNL, especialmente con sede en la Facultad de Humanidades y Ciencias, consistió en entrevistas a los legisladores que elevaron proyectos de ley; generación de documentos y de un informe final que fue elevado al Ministerio de Educación Provincial. El documento elaborado recomienda: ${ }^{(23)}$

Un sistema educativo satisfactorio se caracteriza por algunos atributos: cobertura, retención, graduación, financiamiento, personal, articulación entre niveles, calidad y otros resultados como los aprendizajes logrados por los alumnos o las calificaciones profesionales de su personal. La educación debe servir para la distribución social del conocimiento, la movilidad social ascendente y el avance de la paz y la civilización. La identificación y formulación clara de los problemas públicos, que describen las limitaciones o fallas en aquellos logros, son una condición virtuosa de todo el sistema institucional. Requiere información y racionalidad, debate, conocimiento histórico, comparación y reflexividad sobre los cambios imperantes y acerca de los futuros deseables. (FHUC, 2017:36)

Siguiendo con la dinámica deliberativa de la ley presentada en la Legislatura, luego del breve festejo oficialista en la cámara baja, el proyecto de ley de Educación generó controversias. El Litoral (17/10/2018) confirma que, si bien la iniciativa había sido votada por unanimidad meses atrás en la Cámara de Diputados, y celebrada por todos los sectores políticos con representación parlamentaria, algunas modificaciones al texto original abrieron una instancia de objeciones:

Desde que el proyecto ingresó a la Cámara de Senadores, comenzaron a oírse voces disidentes que no habían cobrado relevancia durante el tránsito del debate en Diputados. Se trata de la iglesia católica, que a través de los obispos de diferentes arquidiócesis, reclamó ser escuchada y planteó objeciones a la media sanción. Los prelados se reunieron,
(22) La cruzada reformista por una nueva carta provincial y el rediseńo integral de una ley que regule la educación provincial, impulsada desde la gestión inaugurada por Miguel Lifschitz (PSFPCyS) constituye un nuevo escenario de debate. Dicho escenario está atravesado por la dinámica propia de los actores gravitantes en el contexto santafesino; por un lado, la legitimidad y respaldo legislativo no está claro que sea posible de consolidar (con más posibilidades en diputados que en senadores). Esto fue imposible en las agendas parlamentarias y la relación de fuerza con las gestiones de los gobernadores que lo antecedieron (Hermes Binner y Antonio Bonfatti) con clara minoría legislativa. Por otra parte, la estructura gremial hegemónica tampoco ha reconocido la tarea como prioridad en la agenda inmediata, es otro factor clave a resolver y el futuro aún es incierto. La Iglesia aún no se ha pronunciado al respecto en modo oficial, sí se ha iniciado un debate académico con las Universidad regionales, UNL y UNR. Y una fuerte convocatoria a la sociedad civil, educadores, especialistas y gobiernos locales en: www. santafe.gob.ar/leyeducacion (23) La comisión de especialistas abordó orientaciones sobre objetivos, propósitos y rol del Estado; gobierno y financiamiento de la educación; igualdad y calidad educativa; educación superior y formación docente. En el documento "Aportes para el debate de una ley de educación en la provincia 
de Santa Fe» se destaca que «los debates y definiciones fundamentales para avanzar en una Ley de Educación podrían ser pensados desde dos aristas: sobre lo estructural; y sobre el contenido». El equipo de docentes e investigadores estuvo conformado por Laura Tarabella, Claudio Lizárraga, Natalia Díaz, Bárbara Mántaras, Nora Grinóvero, Julia Bernik, Marcelino Maina, Victoria Baraldi, Mariela Coudannes, María Laura Sabas, Emanuel Ferreyra,

Marcel Blesio, Sofía Cattáneo, Gabriela Andretich, Andrea Hernán y Adolfo Stubrin.

Este grupo interdisciplinario de especialistas ya habían presentado, en 2017, un primer informe comparativo de los diferentes proyectos elaborados en el ámbito legislativo, así como de la normativa vigente. (24) El Litoral (17/Io/20I8) p. 4

(25) Un día después, el II/IO/20I8 El Litoral informa sobre el encuentro entre referentes de la iglesia y los senadores justicialistas alrededor de estas objeciones. «... miembros de la Iglesia Católica fueron recibidos por varios de los in senadores que conforman el bloque del PJ. Tal como antes se reunieron con legisladores radicales, ahora fue el turno de la bancada mayoritaria. Asistieron monseñor Eduardo Martín, arzobispo de Rosario y presidente de la Comisión de Educación de la Conferencia Episcopal; monseñor Gustavo Help, obispo de Venado Tuerto; presbítero José Luis Ayala, delegado episcopal para la Educación; y el presbítero Osvaldo Macerola. También Javier Bilbao y Walter Van primero, con los senadores del bloque de senadores del Frente Progresista; luego, con los del justicialismo que tiene mayoría en la Cámara. ${ }^{(24)}$

Allí, plantearon su discrepancia y hablaron de un «acuerdo» que no fue tenido en cuenta a la hora de votar la ley. ${ }^{(25)}$

Más allá de la intención del Ejecutivo de buscar consensos a través del diálogo, el proyecto original sufrió aportes importantes en el debate de la cámara de diputados lo que generó polémicas y resistencias. Algunos colegios confesionales de la ciudad distribuyeron un documento a su alumnado emitido por el Consejo Superior de Educación Católica (CONSUDEC). ${ }^{(26)} \mathrm{EI}$ escrito plantea que dicha reforma «con inclusión de ideología de género como obligatoria en las escuelas es un intento más (...) para que sea el estado el que legisle sobre la moral del ciudadano».(27)

\section{Prospectivas}

Con la inquietud y la convicción que estamos ante un escenario en permanente cambio y con posibles novedades a futuro en lo que respecta a este tema entendemos que es viable generar una serie de consideraciones y prospectivas a modo de cierre del presente artículo:

a. Los debates pasados, nacionales y provinciales, y los que vendrán claramente verán nuevamente presentes las presiones corporativas por fuera de los debates legislativos que exponen la larga tradición de estas prácticas políticas que se caracteriza por su dinámica extrainstitucional y habitualmente desconocedora de los parámetros de definición legislativos que combinan, en una república democrática: legalidad y legitimidad.

b. Lo anterior no insta a desconocer las mencionadas voces (gremios, prensa, ONG, iglesia), pero señala la necesidad de propiciar su visibilización pues las corporaciones -desde las religiosas a las gremiales- se caracterizan por una forma de hacer política que consiste en negar que se está haciendo política. Anulando así la posibilidad deliberativa, matriz última de la invención democrática en términos sustantivos. 
c. La licuación de las configuraciones partidarias tradicionales y el predominio de alianzas y coaliciones de lugar a una imprescindible recuperación y análisis de las culturas políticas prevalecientes y la sedimentación de tradiciones que tienden, nocivamente, a naturalizarse.

d. La invención democrática, una agenda progresista que ponga en prioridad los temas y dilemas del siglo XXI, y el debate en torno al decisionismo democrático deben ser aspectos propios del futuro debate en torno a una nueva ley de Educación.

e. El reformismo, polisémica expresión ampliamente disputada por diferentes tradiciones políticas, conlleva varios debates a su interior: uno de ellos es si estamos ante un verdadero sistema educativo -aspecto tratado en el artículo-; otro son las novedades en el campo cultural y de los nuevos derechos que debe incorporarse a esta dinámica.

Inacabado recorrido que hoy presentamos y que anima dos intereses: el abordaje académico de las culturas políticas y los itinerarios educativos en clave del reformismo a escala provincial y, por otro lado, el impacto de estos debates en la invención de una democracia verdaderamente sustantiva.
Meegrot, según lo que se informó oficialmente. Los recibieron el presidente del bloque Armando Traferri, y sus pares José Baucero, Osvaldo Sosa, Ricardo Kaufmann, Joaquín Gramajo, Alcides Calvo, Rubén Pirola, Guillermo Cornaglia y Eduardo Rosconi.» (26) El texto está fechado en setiembre de 2018 en Ciudad Autónoma de Buenos Aires, y fija postura sobre las reformas que intentan introducirse a la ley de Educación Sexual Integral (ESI) a nivel nacional. (27) El Litoral, I7/Io/2018, p. 7

\section{Referencias bibliográficas}

Arata N. y Casanello C. (2006). "Para una mirada genealógica de los Atlas de la educación. El informe Ramos: política, educación y territorio en la Historia de la Instrucción Primaria de la República Argentina», ponencia presentada en las XV Jornadas Argentinas de Historia de la Educación. La Plata (mimeo).

Ascolani, A. (1999). (comp.): La educación en Argentina. Estudios de Historia, Rosario: Ediciones del Arca. Ascolani, A. (2001). "La Historia de la Educación Argentina y la Formación Docente. Ediciones y demanda institucional», en Revista Brasileira de História da Educação/ SBHE No 1.

Beech, J. (2003). «Docentes del Futuro: La influencia de las Agencias Internacionales en las reformas de Formación Docente en Argentina y Brasil en los 90» en Lázaro Lorente, L. y Martínez Usarralde, M. (Eds) (2003). Lecturas de Educación Comparada Y Educación Internacional, 227-255, Valencia: Servei de Publicacions de la Universitat de Valencia.

Bourdie, Pierre y Wacquant, Loic (1995). Respuestas. Por una antropología reflexiva, Grijalbo, México, pp. 229.

Braslavsky, C. (1980). La educación argentina (195580). El País de los Argentinos, Buenos Aires: Centro Editor de América Latina.

Cantero, Adriana (2008): «La ley de Educación Nacional 26.206 y el desafío de la obligatoriedad extendida», en VII Congreso Internacional de Educación, Buenos Aires, Santillana.

Carrizo, B.; Giménez, J.C. (2017). La política en las tramas educativas, Paraná: Editorial La Hendija. 
Cirigliano, G. (1969). Educación y política: el paradojal sistema de la educación argentina. Buenos Aires: Librería del Colegio.

Cucuzza, H. R. (1996). (comp.): Historia de la educación en debate. Buenos Aires: Miño y Dávila.

Chartier, A. M. (2008). "¿Con qué historia de la educación debemos formar a los docentes?» en Anuario de Historia de la Educación No 9, Buenos Aires: SAHE/ Prometeo.

Dupuy, R., (2014). Manual de derecho administrativo laboral docente. Normativa vigente en el sistema educativo provincial. Edición compilada y comentada. Santa Fe, Jurídica Panamericana, segunda edición.

Ginestet, M., Meschiany, T. y Paredes, L. (2007). «Travesías historiográficas en la historia de la educación. Balances y perspectivas en panorámica, ponencia presentada en el VIII Congreso Iberoamericano de Historia de la Educación Latinoamericana (mimeo).

Paviglianiti, N. (1993). "Aproximaciones al desarrollo histórico de la política educacional», Buenos Aires: Oficina de Publicaciones de la Facultad de Filosofía y Letras-UBA.

Popkewitz, T. (1994). Política, conocimiento y poder. Algunas cuestiones para el estudio de las reformas educativas. Universidad de Wisconsin Madison, Revista de Educación № 305.

Popkewitz, T. (2000). Sociología política de las reformas educativas. Tercera Edición (1ra. Ed.: 1994), Madrid: Morata.

Puiggrós, A. (1997). «Espiritualismo, normalismo y educación», en Puiggrós, A. (dir.) Dictaduras y utopías en la historia reciente de la educación argentina. Buenos Aires: Galerna.
Puiggrós, A. (2002). ¿Qué pasó en la educación argentina? Desde la conquista hasta el presente. Buenos Aires: Galerna.

Rivas, Axel (2004): Gobernar la educación. Estudio comparado sobre el poder y la educación en las provincias argentinas, Buenos Aires: Granica.

Rivas, A. [et. al.]: Los Estados provinciales frente a las brechas socio-educativas. Una sociología política de las desigualdades educativas en las provincias argentinas, CIPPEC: Buenos Aires.

Rodríguez, L. G. (2013). «La educación en los últimos treinta años» en Cuestiones de Sociología (9), 305310. En Memoria Académica. Disponible en:http:// www.memoria.fahce.unlp.edu.ar/art_revistas/pr.5896/ pr.5896.pdf

Russen, J. (1992). "El desarrollo de la competencia narrativa en el aprendizaje histórico. Una hipótesis ontogenética relativa a la conciencia moral», en Propuesta Educativa No 7, Buenos Aires: FLACSO-Miño y Dávila.

Schmucler, H. (1990). «Innovación de la política cultural en la Argentina» en AA.VV., ¿Hacia un nuevo orden estatal en América Latina?, Innovación cultural y actores socioculturales, Argentina, Buenos Aires, Clacso, Biblioteca de Ciencias Sociales.

Suasnabar, C. (2004). Universidad e Intelectuales. Educación y política en Argentina (1955-1966). Buenos Aires: FLACSO Manantial.

Weinberg, G. (1985). Modelos educativos en la historia de América Latina. Buenos Aires: Kapelusz. 